\title{
Diagnostic Accuracy of Neurological Problems in the Emergency Department
}

\author{
Jeremy J Moeller, Joelius Kurniawan, Gordon J Gubitz, \\ John A Ross, Virender Bhan
}

\begin{abstract}
Background: Previous studies describe significant rates of misdiagnosis of stroke, seizure and other neurological problems, but there are few studies examining diagnostic accuracy of all emergency referrals to a neurology service. This information could be useful in focusing the neurological education of physicians who assess and refer patients with neurological complaints in emergency departments. Methods: All neurological consultations in the emergency department at a tertiary-care teaching hospital were recorded for six months. The initial diagnosis of the requesting physician was recorded for each patient. This was compared to the initial diagnosis of the consulting neurologist and to the final diagnosis, as determined by retrospective chart review. Results: Over a six-month period, 493 neurological consultations were requested. The initial diagnosis of the requesting physician agreed with the final diagnosis in $60.4 \%$ (298/493) of cases, and disagreed or was uncertain in $35.7 \%$ of cases $(19.1 \%$ and $16.6 \%$ respectively). In $3.9 \%$ of cases, the initial diagnosis of both the referring physician and the neurologist disagreed with the final diagnosis. Common misdiagnoses included neurocardiogenic syncope, peripheral vertigo, primary headache and psychogenic syndromes. Often, these were initially diagnosed as stroke or seizure. Conclusions: Our data indicate that misdiagnosis or diagnostic uncertainty occurred in over one-third of all neurological consultations in the emergency department setting. Benign neurological conditions, such as migraine, syncope and peripheral vertigo are frequently mislabeled as seizure or stroke. Educational strategies that emphasize emergent evaluation of these common conditions could improve diagnostic accuracy, and may result in better patient care.
\end{abstract}

RÉSUMÉ: Exactitude du diagnostic des problèmes neurologiques à la salle d'urgence. Contexte : Certaines études font état d'un taux significatif de diagnostics erronés d'accidents cérébrovasculaires, de crises convulsives et d'autres problèmes neurologiques, mais peu d'études ont examiné l'exactitude diagnostique de tous les cas référés d'urgence à un service de neurologie. Cette information serait utile pour cibler l'éducation neurologique des médecins qui évaluent et qui réfèrent les patients qui consultent à la salle d'urgence pour des troubles neurologiques. Méthodes : Toutes les consultations neurologiques à la salle d'urgence d'un hôpital d'enseignement de soins tertiaires ont été relevées sur une période de six mois. Le diagnostic initial du médecin référant a été noté pour chaque patient. Une revue rétrospective de dossiers a permis de comparer ce diagnostic au diagnostic initial du neurologue consultant et au diagnostic final. Résultats : Au cours d'une période de six mois, 493 consultations ont été demandées en neurologie. Le diagnostic initial du médecin référant était concordant avec le diagnostic final chez 60,4\% des cas (298/493) et discordant ou incertain chez $35,7 \%$ des cas $(19,1 \%$ et 16,6\% respectivement). Chez 3,9\% des cas, le diagnostic initial du médecin référant et du neurologue ne concordaient pas avec le diagnostic final. Les diagnostics erronés les plus fréquents étaient la syncope neurocardiogénique, le vertige d'origine périphérique, la céphalée primaire et les syndromes psychogéniques. Souvent ces cas recevaient un diagnostic initial d'un accident cérébrovasculaire ou de crise convulsive. Conclusions : Nos données révèlent qu'un diagnostic erroné ou incertain a été posé chez plus du tiers des patients vus à la salle d'urgence qui sont référés en neurologie. Des affections neurologiques bénignes comme la migraine, la syncope et le vertige d'origine périphérique sont fréquemment diagnostiqués comme une crise convulsive ou un accident cérébrovasculaire. Des stratégies d'éducation qui mettent l'emphase sur l'évaluation de ces affections fréquentes à la salle d'urgence pourraient améliorer l'exactitude diagnostique ainsi que les soins prodigués aux patients.

Can. J. Neurol. Sci. 2008; 35: 335-341

Neurological disorders in the emergency department (ED) setting are diverse in presentation and diagnostic spectrum. At least $6 \%$ of all emergency department visits are for neurological problems. ${ }^{1}$ An important issue is that of diagnostic accuracy. Previous retrospective analyses in the neurological literature have demonstrated that $5-11 \%$ of all patients given the diagnosis of stroke in the emergency department had another diagnosis altogether. ${ }^{2-4}$ Similarly, $13-26 \%$ of patients referred to epilepsy
From the Division of Neurology, Department of Medicine (JJM, JK, GJG, VB), Department of Emergency Medicine (JAR), Queen Elizabeth II Health Sciences Centre and Dalhousie University, Halifax, Nova Scotia, Canada.

Received August 20, 2007. Final Revisions Submitted January 25, 2008. Reprint requests to: Jeremy Moeller, Room 3850, Halifax Infirmary Site, Queen Elizabeth II Health Sciences Centre, 1796 Summer Street, Halifax, Nova Scotia, B3H 3A7, Canada. 
clinics were found not to have epilepsy. ${ }^{5-7}$ These misdiagnoses may have major implications on the medical care provided to patients, as well as impacting on their quality of life. Such errors can also reduce efficiency within the health care system.

Although there are many studies that investigate diagnostic accuracy in particular sub-fields within neurology, we are aware of only one study that reported the diagnostic accuracy of all referrals made to a general neurology service. ${ }^{8}$ This study is not likely reflective of Canadian ED practice, as $14.7 \%$ of all patients admitted to the emergency department had a formal neurological consultation.

Identifying patterns of diagnostic uncertainty could have major implications for patient care, as well as for neurological education. Although similar data has not been published for Canadian medical schools, a recent review of U.S. medical schools reported that only $80 \%$ of medical schools require clinical experience in neurology, and in most schools, this consists of a two-week rotation in fourth year. ${ }^{9}$ Only $17.4 \%$ of emergency medicine residency programmes in the U.S. require a neurology rotation, and only 12.0 hours of didactic neurology teaching was provided in these programmes annually. ${ }^{10}$ With neurology education time being so limited, care must be taken to identify the areas in which we should concentrate the most educational resources.

The aim of this study was to identify the most commonly misdiagnosed neurological problems in the ED. By doing this, we may help to optimize neurological education for nonneurologists, especially those who encounter patients with emergent neurological problems.

\section{Methods}

\section{Search Strategy and Literature Retrieval}

With the assistance of a qualified health librarian, we performed a PubMed search using all combinations of the search terms and MeSH headings "Nervous System Diseases/ diagnosis" with "misdiagnosis, Diagnostic Errors, diagnostic accuracy" and "Emergency Medical Services, Family Physicians, primary care." We also searched the Cochrane Library using the same search strategy. After review of the titles and abstracts, we identified 25 articles that investigate diagnostic accuracy of neurological problems by primary care or ED physicians. A review of the reference lists did not reveal any new relevant publications. Most articles focused on the diagnosis of specific neurological conditions. Only one article ${ }^{8}$ reviewed all patients seen by a neurology service in the ED. As mentioned in the introduction, this study differs considerably from ours in both purpose and design.

\section{Study Site}

The Queen Elizabeth II Health Sciences Centre is a quaternary-care, university-affiliated hospital in Halifax, Nova Scotia, Canada. It provides core health care for a population of 350,000 , and is the major tertiary referral centre for the province of Nova Scotia. It is an adult hospital, providing care to patients aged 16 years and older.

The Neurology Service provides a 24 hour consultation service for the main urban Emergency Department, as well as neurological support to emergency departments, primary care physicians and specialists around the province of Nova Scotia. Referring physicians have a number of options when consulting a neurologist on an emergency basis. In some cases, the consultation is limited to telephone advice, while in others, patients can be referred to out-patient clinics on an urgent or semi-urgent basis. When an outside referring physician determines that the patient requires emergent neurological evaluation, a transfer to the emergency department at our institution can be arranged through the neurologist on call. Emergency physicians within our hospital also have the option of directly consulting the neurology resident on call. Trainees (Residents and Clerks) within the emergency department also have the option of direct consultation, but only after reviewing the patient with the supervising emergency physician. Every patient seen by a neurology resident in the emergency department is reviewed by a staff neurologist.

The protocol for this study was approved by the institutional Research Ethics Board.

\section{Subject Selection}

As part of routine clinical care and record-keeping, the neurology residents at the research site maintained a prospective 'on-call log', recording information about all patients seen in the ED. These patients were referred from one of three sources: (1) directly from an emergency physician or emergency department trainee (clinical clerk or resident); (2) emergent referral from a local family physician; or, (3) transfer from another hospital within the province for further evaluation and management. Along with the date, a record was made of the name of the resident and staff neurologist on-call, the patient's name, date of birth and hospital unit number, the diagnosis made by the referring physician, the initial neurological diagnosis, and the disposition of the patient (e.g. admitted to hospital, out-patient follow-up, etc.). The medical record was also used to determine the length of stay in the emergency department for patients who were discharged directly from the ED, and duration of stay in hospital for patients who were admitted.

\section{Study Period}

We analyzed all emergent neurological consultations from December 29, 2005 to July 1, 2006.

\section{Diagnostic Accuracy}

The initial diagnosis of the referring physician was compared to a final diagnosis, which was determined by retrospective chart review. The definition of "final diagnosis" depended upon the final disposition of the patient: for patients who were admitted, the discharge diagnosis was used; for patients who were seen in follow-up by a neurologist, the final diagnosis was determined from the consultation letter; for patients who were discharged home from the emergency department with no follow-up, the neurologists' diagnosis in the emergency department record was used. In all cases, the diagnosis was based upon an interpretation of history, physical examination and appropriate diagnostic tests.

The diagnostic accuracy of the referring physician was divided into three major categories: "agree," "disagree," and "uncertain." The referring physician's diagnosis was determined as "agreed" when the initial diagnosis was consistent with the 
final diagnosis. When the referring physician did not suggest a diagnosis, but rather referred the patient with unexplained symptoms (i.e. "weakness not yet diagnosed"), we defined this as "uncertain." The referring physician's diagnosis was deemed as "disagreed" if the final diagnosis was completely different.

We also compared the initial neurological diagnosis to the final diagnosis, excluding those cases when the final diagnosis was defined as the initial neurological diagnosis. The same definitions of "agree", "disagree" and "uncertain" were used. We used the designation of "both disagree" when the initial diagnosis of both the referring physician and the consulting neurologist was different from the final diagnosis.

\section{Statistical Analysis}

Categorical variables were compared using Chi-squared analysis. Continuous variables were compared using either unpaired t-test (for dichotomous variables) or one-way analysis of variance (ANOVA) for comparisons between three or more groups. Ninety-five percent confidence intervals were calculated assuming a normal distribution around the mean.

\section{RESULTS}

\section{Number of Consultations}

Residents completed the prospective "on-call log" for 165 $(90 \%)$ of a possible 184 on-call shifts during the study period. There was no significant difference in the rate of record-keeping from month to month $(\mathrm{p}=\mathrm{NS})$. Consultations were recorded, 493 during this study period, which represented a mean of 2.9 (2.73.2) consultations per call shift.

\section{Diagnostic Accuracy of Referring Physician}

The initial diagnosis of the referring physician agreed with the final diagnosis $60.4 \%$ (298/493) of the time. Of the remaining $39.6 \%$ of consultations, the initial diagnosis of the referring physician disagreed in $19.1 \%$ (94/493) of cases, while there was significant uncertainty in $16.6 \%$ (82/493) of referrals. In $3.9 \%(19 / 493)$ of cases, the initial diagnosis of the referring physician agreed with the initial neurological diagnosis, but both disagreed with the final diagnosis. The characteristics of each referral are shown in Table 1. There was no significant difference in diagnostic accuracy based on patient gender or level of training of the neurology resident $(\mathrm{p}=\mathrm{NS})$. However, the patients in whom there was initial diagnostic uncertainty were significantly younger than the other groups $(\mathrm{p}=0.001)$.

The final diagnosis was determined using hospital records (discharge or death summary) in $333(68 \%)$ cases, while outpatient follow-up records were used in $95(19 \%)$ cases. In the remaining $65(13 \%)$ cases, the neurologist's diagnosis in the emergency department record was used. Of the 65 patients for whom the final diagnosis was determined in the emergency department, $11(17 \%)$ had migraine, $8(12 \%)$ had peripheral vestibulopathy, 6 (9\%) had syncope, and $13(20 \%)$ had a variety of other neurological syndromes. Six patients $(9 \%)$ had a psychiatric cause for their symptoms and $14(22 \%)$ had a primary medical, non-neurological cause for their symptoms. The remaining 7 patients $(10 \%)$ were diagnosed with minor stroke or transient ischemic attack (TIA) in the emergency department, discharged home, and then lost to follow-up.

Referrals were from emergency physicians in 60\% (267/493) of cases. An additional 22\% (108/493) of cases were from

Table 1: Characteristics of neurological consultations seen in the Emergency Department based upon the diagnostic accuracy of the referring physician

\begin{tabular}{|c|c|c|c|c|c|}
\hline Characteristic & $\begin{array}{c}\text { Agree } \\
(\mathrm{n}=\mathbf{2 9 8})\end{array}$ & Uncertain $(\mathrm{n}=\mathbf{8 2})$ & Disagree $(n=94)$ & $\begin{array}{c}\text { Both Disagree } \\
(n=19)\end{array}$ & $p$ value \\
\hline Mean Pt Age (CI) & $64.1(61.9-66.2)$ & $54.1(49.5-58.7)$ & $59.9(56.1-63.7)$ & $61.4(53.5-69.4)$ & $0.001^{*}$ \\
\hline Median Pt Age (range) & $66(16-104)$ & $55(16-86)$ & $62(18-96)$ & $59(26-92)$ & \\
\hline Female, n (\%) & $169(58 \%)$ & $47(57 \%)$ & $48(51 \%)$ & $10(53 \%)$ & $0.16^{* *}$ \\
\hline \multicolumn{6}{|l|}{$\begin{array}{l}\text { Referring Physician, } \mathbf{n} \\
(\%)\end{array}$} \\
\hline $\mathrm{EP}$ & $177(66 \%)$ & $29(11 \%)$ & $48(17 \%)$ & $13(5 \%)$ & \\
\hline ED Trainee & $62(57 \%)$ & $23(21 \%)$ & $20(19 \%)$ & $3(3 \%)$ & \\
\hline Family Physician & $19(45 \%)$ & $13(31 \%)$ & $9(21 \%)$ & $1(2 \%)$ & \\
\hline External/Other & $40(53 \%)$ & $17(22 \%)$ & $17(22 \%)$ & $2(3 \%)$ & $0.02 * *$ \\
\hline
\end{tabular}


Table 2: Initial diagnosis of referring physician compared to the final neurological diagnosis in cases in which there was significant disagreement $(n=94)$
Initial Diagnosis of the Referring Physician (n)

Stroke or TIA (67)

\author{
Final Diagnosis (n)* \\ Psychogenic symptoms (11) \\ Peripheral vestibulopathy (9) \\ Migraine with aura (9) \\ General Medical Condition \\ Metabolic encephalopathy (6) \\ Syncope (5) \\ Other cardiovascular condition (4) \\ Musculoskeletal injury (2) \\ Concussion/Trauma (4) \\ Peripheral neuropathy/radiculopathy (4) \\ Brain Tumour (3) \\ Transient global amnesia (2) \\ Bell's palsy (1) \\ Seizure (1) \\ Medically unexplained symptoms (6) \\ Syncope (6) \\ Medically unexplained symptoms (1) \\ Benign headache syndrome (3) \\ Transverse myelitis (1) \\ Subarachnoid hemorrhage (1) \\ Musculoskeletal injury (1)
}

Other $(15)^{* *}$

in which there was agreement between the initial and final diagnoses. The rate of stroke/TIA or seizure is more than tenfold higher in the "agree" group compared to the "disagree" group $(75.4 \%$ vs $6.4 \%, \mathrm{p}<0.001)$.

\section{Diagnostic Accuracy of Consulting Neurologist}

In the 428 cases where the final diagnosis was determined from sources other than the neurologist's emergency department record, the consulting neurologist's initial diagnosis agreed with the final diagnosis in $344(80 \%)$ cases. In $65(15 \%)$ cases, there was uncertainty, and in $19(4.4 \%)$ cases, the initial diagnosis of the neurologist disagreed with the final diagnosis. In all 19 cases, the initial diagnosis of the referring physician also disagreed with the final diagnosis, and so these were categorized as "both disagree."

In the "both disagree" cases, there was a trend toward the initial, incorrect diagnoses of either stroke/TIA $(16 / 19 ; 84.2 \%)$ or seizure $(2 / 19 ; 10.5 \%)$. In the cases where stroke was

Table 3: Initial symptoms or signs and final diagnosis in patients referred for neurological consultation for whom the referring physician did not propose an etiology $(n=82)$

* Final diagnosis is defined as the best available option of: (i) discharge diagnosis from hospital, (ii) diagnosis from consultant's followup letter, or (iii) neurologist's diagnosis in the emergency department; ** This represents cases in which the particular initial diagnosis was made only once; TIA=transient ischemic attack.

emergency department trainees. The remaining referrals were from family physicians or physicians in other hospitals. Emergency physicians were significantly more likely to agree with the final diagnosis than other referral sources (Table 1; $\mathrm{p}<0.02$ ), and significantly less likely to express diagnostic uncertainty. Rates of disagreement with the final diagnosis were similar in all physician groups.

In the cases where the initial impression of the referring physician disagreed with the final neurological diagnosis, there was a pattern of incorrect diagnosis of stroke and seizure. Of the 94 "disagree" patients, the initial diagnosis was stroke or seizure in $71 \%(67 / 94)$ and $7 \%(7 / 94)$ of cases, respectively. The final diagnoses were frequently "benign" conditions such as peripheral vestibulopathy, migraine, and neurocardiogenic syncope. Other medical conditions and psychiatric syndromes were also frequently interpreted as stroke or seizure by the referring physician (Table 2).

In cases where the referring physician was uncertain of the diagnosis, the final diagnoses were heterogeneous, and included a broad variety of neurological, psychiatric and general medical conditions. Notably, only 7\% (6/82) of patients were diagnosed with a stroke syndrome. After a complete neurological work-up, $26 \%(21 / 82)$ of patients were left with no definitive diagnosis (Table 3).

When the final diagnosis was the same as the initial diagnosis of the referring physician, the most common syndromes were stroke/TIA $(192 / 298 ; 64.4 \%)$ and seizure $(34 / 298 ; 11.0 \%)$ (Table 4). Together, these syndromes represented $75.4 \%$ of all referrals

\begin{tabular}{|c|c|}
\hline Unexplained Symptoms or Signs (n) & Final Diagnosis (n) \\
\hline \multirow[t]{8}{*}{ Focal Weakness (17) } & Migraine (2) \\
\hline & Psychogenic weakness (2) \\
\hline & Stroke (1) \\
\hline & Spinal cord syndrome (2) \\
\hline & Guillain-Barre syndrome (1) \\
\hline & Focal neuropathy (1) \\
\hline & Arthritis (1) \\
\hline & Medically unexplained symptoms ( 7$)$ \\
\hline \multirow{7}{*}{ Decreased level of consciousness or confusion (17) } & Metabolic encephalopathy (5) \\
\hline & Psychogenic symptoms (5) \\
\hline & Stroke/TIA (2) \\
\hline & Seizure (2) \\
\hline & Syncope (1) \\
\hline & Creutzfeldt-Jakob Disease (1) \\
\hline & Medically unexplained symptoms (1) \\
\hline \multirow[t]{5}{*}{ Sensory disturbance, including pain (15) } & Migraine (4) \\
\hline & Spinal cord syndrome (4) \\
\hline & Peripheral neuropathy (3) \\
\hline & Stroke (1) \\
\hline & Medically unexplained symptoms (3) \\
\hline \multirow[t]{5}{*}{ Ataxia and/or Gait Disturbance (7) } & Metabolic derangement (2) \\
\hline & Concussion (1) \\
\hline & Multiple sclerosis (1) \\
\hline & Guillain-Barre syndrome (1) \\
\hline & Medically unexplained symptoms (2) \\
\hline \multirow[t]{2}{*}{ Headache (7) } & Primary headache syndrome (6) \\
\hline & Brain tumour (1) \\
\hline \multirow[t]{4}{*}{ Visual disturbance (6) } & Viral encephalitis (1) \\
\hline & Stroke (1) \\
\hline & Fourth nerve palsy (1) \\
\hline & Medically unexplained symptoms (3) \\
\hline \multirow[t]{3}{*}{ Dizziness/Vertigo (5) } & Peripheral vestibulopathy (3) \\
\hline & Stroke (1) \\
\hline & Medically unexplained symptoms (1) \\
\hline \multirow[t]{5}{*}{ Abnormal neurological examination* (8) } & Brain tumour (1) \\
\hline & Creutzfeldt-Jakob Disease (1) \\
\hline & Spinal cord syndrome (1) \\
\hline & Psychogenic syndrome (1) \\
\hline & Medically unexplained signs (4) \\
\hline
\end{tabular}

*Abnormal examination findings included tremor, myoclonus, aphasia and multifocal signs 
Table 4: Final diagnosis for all patients for whom the initial diagnosis of the referring physician agreed with the final neurological diagnosis $(n=298)$.

\begin{tabular}{lc} 
Diagnosis & Number of Patients (\%) \\
Stroke/TIA & $192(64)$ \\
Seizure & $34(11)$ \\
Primary Headache Syndome & $13(4)$ \\
Other Neurological Syndromes & $43(14)$ \\
Other Non-neurological Syndromes & $16(5)$ \\
\hline
\end{tabular}

mistakenly diagnosed, the final diagnoses included other general medical conditions $(5 / 16 ; 31.3 \%)$, other chronic neurological conditions $(3 / 16 ; 18.8 \%)$, conversion disorder $(2 / 16 ; 12.5 \%)$, and glioblastoma multiforme $(1 / 16 ; 6.3 \%)$. For the remaining five patients initially diagnosed with stroke/TIA, no clear diagnosis could be made. Of the two patients initially diagnosed with seizure, one was later determined to have psychogenic pseudoseizures, while the other was eventually diagnosed with neurocardiogenic syncope. In both cases, video electroencephalography was used to make the diagnosis. One patient was initially diagnosed with migraine and was later determined to have a patent foramen ovale with multiple sub-cortical cerebral infarctions.

\section{Disposition}

The disposition of patients after being evaluated in the ED differed significantly depending upon the diagnostic accuracy of the referring physician (Table 5). When there was either diagnostic agreement or uncertainty, the majority of patients (72\% and $73 \%$, respectively) were admitted to hospital for treatment and/or further investigation. In comparison, significantly fewer patients in whom there was diagnostic disagreement were admitted to hospital $(32 \%, \mathrm{p}<0.001)$, and a significantly greater number were either discharged home with no follow-up $(27 \%$ vs $5 \%$ in agree/uncertain, $\mathrm{p}<0.001)$ or referred to another service or back to the consulting physician ( $14 \%$ vs $3 \%$ (agree) vs $10 \%$ uncertain, $\mathrm{p}<0.001$ ).

\section{Time in the Emergency Department and Length of Stay in} Hospital

As shown in Table 5, patients in whom there was significant diagnostic uncertainty spent significantly longer in the emergency department than patients in the "agree" and "disagree" categories (10.2 hours vs. 7.3 and 7.8 hours, respectively, $\mathrm{p}=0.02$ ). Among patients admitted to hospital, those in whom there was agreement between the referring physician and the final diagnosis had a significantly longer hospital stay, 27.0 days, compared to less than 10-day stays for all other groups $(\mathrm{p}=0.005)$. When patients with the final diagnosis of stroke were excluded, the mean duration of stay in the "agree" group decreases to 17.0 days, and there was no significant difference between groups $(\mathrm{p}=0.06)$.

\section{Discussion}

In almost five hundred emergency neurological consultations over a six-month period, there was diagnostic uncertainty in over one-third of patients. In one-fifth of patients, a diagnosis was proposed that was much different from the final neurological diagnosis. In these cases, syndromes such as headache, peripheral vestibulopathy, psychiatric syndromes and other

Table 5: Disposition and time in emergency department and/or as inpatient, based on diagnostic accuracy

\begin{tabular}{|c|c|c|c|c|c|}
\hline & $\begin{array}{c}\text { Agree } \\
(n=298)\end{array}$ & Uncertain $(n=82)$ & Disagree $(n=94)$ & $\begin{array}{c}\text { Both Disagree } \\
\quad(n=19)\end{array}$ & $p$ value \\
\hline \multicolumn{6}{|l|}{ Disposition } \\
\hline Admitted & $216(73 \%)$ & $60(73 \%)$ & $31(33 \%)$ & $18(95 \%)$ & \\
\hline OP Follow-up & $59(20 \%)$ & $10(12 \%)$ & $25(27 \%)$ & $1(5 \%)$ & \\
\hline No neuro $\mathrm{f} / \mathrm{u}$ & $14(5 \%)$ & $4(5 \%)$ & $25(27 \%)$ & 0 & \\
\hline Other & $9(3 \%)$ & $8(10 \%)$ & $13(14 \%)$ & 0 & $<0.001$ \\
\hline $\begin{array}{l}\text { Length of stay, } \\
\text { days (CI) }\end{array}$ & $27.0(20.2-33.8)$ & $9.6(7.4-11.7)$ & $8.7(5.1-12.4)$ & $9.6(4.9-14.3)$ & 0.005 \\
\hline $\begin{array}{l}\text { Time in ED, hours } \\
\text { (CI) }\end{array}$ & $7.3(6.6-7.9)$ & $10.3(6.4-14.1)$ & $7.8(6.9-8.7)$ & $\mathrm{N} / \mathrm{A}$ & 0.02 \\
\hline
\end{tabular}

$\mathrm{OP}=$ out-patient, $\mathrm{f} / \mathrm{u}=$ follow-up, $\mathrm{ED}=$ emergency department, $\mathrm{CI}=95 \%$ confidence interval, N/A = not applicable

"Agree" represents consultations in which the initial diagnosis of the referring physician agrees with the final neurological diagnosis, "Disagree" is when the referring diagnosis is different than the final diagnosis, "Uncertain" is when no initial diagnosis was proposed, and "Both Disagree" is when the initial diagnosis of both the referring physician and the neurologist is different than the final diagnosis 
medical problems were most often initially diagnosed as stroke. In many instances, the final diagnosis was made without any additional diagnostic tests. In particular, migraine syndromes and peripheral vestibulopathy could be distinguished from stroke on the basis of symptoms, signs and normal computed tomography of the brain (ordered in the ED prior to consultation). Similarly, neurocardiogenic syncope was often distinguished from a seizure or TIA on the basis of history alone. This might suggest that an educational intervention (in medical school or residency training or as continuing medical education in emergency departments) could provide primary care physicians with the skills to make the distinction between stroke or seizure and more benign "mimics" in the ED or doctor's offices.

There was a significantly higher degree of agreement with the final diagnosis in referrals from emergency physicians than in referrals from trainees, family physicians and outside hospitals. Much of this is reflected in the fact that the three latter referral sources displayed a higher degree of diagnostic uncertainty. A possible explanation for this is that emergency physicians have significantly more experience with acute neurological problems; patients with stroke and seizure are far less likely to present first to a family physician's office. Additionally, there may be a referral bias. Family physicians and physicians at outside hospitals are more likely to refer patients with the most difficultto-diagnose problems for emergent neurological evaluation. Patients with more routine problems could be referred directly to outpatient clinics, or specialized advice could be provided over the telephone.

Further evidence supporting the argument that accurate early recognition of "benign" diagnoses may save health care resources comes from the eventual destination of patients seen in neurological consultation. In the groups where there was uncertainty, and when the initial diagnosis agreed with the final diagnosis, almost three-quarters of patients were admitted to hospital. This suggests that these patients required urgent treatment and/or diagnostic tests. In comparison, less than a third of patients in whom the final diagnosis was different than the initial diagnosis were admitted to hospital, possibly suggesting that, overall, these patients might require less urgent attention.

Patients referred for urgent neurological consultation spend an average of almost eight hours in the ED. This time is significantly longer for patients in whom there is initial diagnostic uncertainty. An educational intervention that assists primary care and emergency physicians in improving diagnostic accuracy might reduce this number, thus reducing the time these patients spend in the ED.

This research demonstrates that stroke syndromes are also over-diagnosed by neurologists in the ED. In almost all of the cases where neurologists' initial diagnosis was different than the final diagnosis, the patients' symptoms and signs were interpreted as stroke. It seems that there is a tendency to interpret acute-onset neurological symptoms and/or signs as stroke. This may be understandable on the basis that stroke was the most common neurological diagnosis in our study population. It is also interesting to note that in only one case was a more "benign" diagnosis (migraine) made for a more serious problem (patent foramen ovale with multiple micro-infarcts). Reassuringly, diagnostic patterns appear to err on the side of patient safety.
Neurological problems present challenges to primary care physicians and neurologists alike. In almost one-fifth of all referrals for neurological consultation, no clear diagnosis was made in the ED. Even after thorough investigation and observation, one-fifth of those cases remained unexplained. This likely reflects the complexity of nervous system disease and the multitude of clinical presentations that can be encountered.

\section{Limitations}

There are a number of potential limitations to this study. Our data represent only patients referred for urgent neurological assessment. This likely represents a small fraction of the patients with neurological problems seen in the ED every day. Assuming that accurate diagnosis is made in all patients who are not referred to a neurologist, our data would over-estimate the rate of diagnostic uncertainty.

Another limitation of these data is the fact that in many cases, the "final" diagnosis was made using only history, physical examination and selected diagnostic tests. For many of these diagnoses, there is no "gold standard," which leaves room for interpretation. When possible, this problem was overcome by reviewing hospital charts for at least six months after the initial consultation, and ensuring that another diagnosis was not made in the interim.

In interpreting the initial diagnosis of the referring physician, we must take into account the fact that, in some cases, only a tentative diagnosis is proposed. Primary care physicians often make 'on the spot diagnoses' for which the strongest consideration is patient safety. Therefore, there may be a tendency to propose the most serious diagnosis, in order that the patient receives more urgent diagnostic testing and specialist attention. Additionally, many neurological syndromes evolve over time. For example, a patient presenting with a visual field defect may initially appear to be having a stroke, and so a neurologist is consulted. However, by the time the neurologist's assessment is complete, the patient may have developed a headache, helping to confirm the diagnosis of migraine. These practical realities of emergency medicine may also over-estimate "misdiagnosis" rates.

\section{Conclusions}

Even when the above considerations are taken into account, we believe that our results present helpful guidance for the neurological education of emergency and primary care physicians. We have identified several diagnostic patterns that consistently cause problems, and in many cases, these problems might be solved with education. Relatively benign neurological conditions, such as migraine, syncope and peripheral vertigo are often labeled as more serious conditions such as seizure or stroke. Educational strategies that stress the emergent evaluation of these common conditions could improve diagnostic accuracy, and may result in better patient care.

\section{ACKNOWLEDGEMENTS}

This study did not receive funding from any source, commercial or otherwise. JM had full access to all the data in the study and takes responsibility for the integrity of the data and the accuracy of the data analysis. 
The authors would like to acknowledge the physicians who participated in this study, particularly the emergency physicians, neurologists and neurology residents at the Queen Elizabeth II Health Sciences Centre in Halifax, Nova Scotia, Canada.

\section{REFERENCES}

1. McCaig LF, Nawar EW. National Hospital Ambulatory Medical Care Survey: 2004 emergency department summary. Adv Data. 2006;372:1-29.

2. Morgenstern LB, Lisabeth LD, Mecozzi AC, Smith MA, Longwell PJ, McFarling DA, et al. A population-based study of acute stroke and TIA diagnosis. Neurology. 2004;62:895-900.

3. Ferro JM, Pinto AN, Falcao I, Rodrigues G, Ferreira J, Falcao F, et al. Diagnosis of stroke by the non-neurologist: a validation study. Stroke. 1998;29:1106-9.

4. Kothari RU, Brott T, Broderick JP, Hamilton CA. Emergency physicians. Accuracy in the diagnosis of stroke. Stroke. 1995;26: 2238-41.
5. Josephson CB, Rahey S, Sadler RM. Neurocardiogenic syncope: frequency and consequences of its misdiagnosis as epilepsy. Can J Neurol Sci. 2007;34:221-4.

6. Smith D, Defalla BA, Chadwick DW. The misdiagnosis of epilepsy and the management of refractory epilepsy in a specialist clinic. Q J Med. 1999;92:15-23.

7. Scheepers B, Clough P, Pickles C. The misdiagnosis of epilepsy: findings of a population study. Seizure. 1998;7:403-6.

8. Moulin T, Sablot D, Vidry E, Belahsen F, Berger E, Lemounaud P, et al. Impact of emergency room neurologists on patient management and outcome. Eur Neurol. 2003;50:207-14.

9. Galetta SL, Jozefowicz RF, Avitzur O. Advances in neurological education: a time to share. Ann Neurol. 2006;59:584-90.

10. Stettler BA, Jauch EC, Kissela B, Lindsell CJ. Neurologic education in emergency medicine training programs. Acad Emerg Med. 2005;12:909-11. 